Aus der Kgl. Univ.-Ohrenklinik zu Halle a. S.

XI.

\title{
Ein neues operatives Verfahren zur Verhütung der Wieder- verwachsung des Hammergriffes mit der Labyrinthwand nach ausgeführter Synechotomie und Tenotomie des $\mathbf{M}$. tensor tympani. ${ }^{1}$ )
}

Von

Priv.-Doc. Dr. med. Grunert.

I. Assistenzarzt der Klinils.

Es ist bekannt, dass unter besonders günstigen Bedingungen die Möglichkeit vorhanden ist, in Fällen von Verwachsung des stark eingezogenen Hammergriffes mit der Labyrinthwand durch Trennung der Adhäsionen in Verbindung mit der Tenotomie des M. tensor tympani eine Hörverbesserung und Beseitigung gewisser subjectiver Beschwerden zu erzielen. Aber darin stimmt die Mehrzahl der Fachgenossen überein, dass der so erzielte Erfolg ein nur sehr temporärer ist. In der Regel sieht man spätestens wenige Wochen nach der Operation den Hammergriff wieder in seiner friiheren Stellung, und Hand in Hand damit den erzielten functionellen Erfolg wieder verschwunden.

Man kann sich dann durch die Untersuchung mit dem Siegle'schen Trichter von dem Wiedereintritt der Beweglichkeitseinschränkung oder Aufhebung des Hammergriffes überzengen, welche auf die Wiederbildung der Adhäsionen bezogen werden muss. $\mathrm{Ob}$ in solchen Fallen ebenfalls eine Wiederverwachsung der Sehnenenden des M. tensor tympani eingetreten ist, entzieht sich zwar dem objectiven Nachweise mittelst der Ohruntersuchungsmethoden und ist meines Wissens zur Zeit auch noch nicht durch die Section von Schläfenbeinen fruther Tenotomirter anatomisch bewiesen, wird aber von der Mehrzahl der Fachcollegen angenommen. Mit Recht hat man in diesem Wieder-

1) Vortrag, gehalten in der Section für Ohrenheilk. der 69. Versammlung deatscher Naturforscher und Aerzte zu Braunschweig. 
eintritt der Verwachsungen die Ursache des Wiederverlustes des ursprïnglichen functionellen Operationserfolges erblickt und mit allen möglichen Mitteln angestrebt, denselben zu verhüten. Alle diese Versuche haben zu einem befriedigenden Resultate nicht geführt; vielfach haben sie vielmehr noch dadurch Schaden angerichtet, dass sie zu einer secundären Infection der Schleimhaut und Ohreiterung fülırten.

Mein Chef, Herr Geheimrath Schwartze, war so freundlich, mich in seiner Klinik in einigen Fällen ein operatives Verfahren erproben zu lassen, mittelst dessen es mir mit Sicherheit gelang, den Eintritt der Wiederverwachsung zu verhüten. Wenn ich nun auch nicht in der Lage bin, Ihnen, meine Herren, einen Bericht über glänzende Erfolge in functioneller Hinsicht bieten zu können, so giebt mir doch der Umstand, dass ich glaube, diese Frage in technischer Hinsicht gelöst zu haben, den Muth, Ihnen kurz uber dieses Verfahren zu berichten. Dasselbe ist folgendes:

Nach Anlegung zweier dem Hammergriff parallel verlaufender und nach oben bis an den Margo tympanicus reichender Schnitte im vorderen oberen und hinteren oberen Trommelfellquadranten durchtrennt man mit dem in den hinteren Schnitt eingefuibrten Schwartze'schen Tenotom in der von Schwartze angegebenen Weise die Tensorsehne. Hierauf zieht man mit der nach unten gerichteten Schneide desselben Tenotoms zwischen Hammergriff und Labyrinthwand hinab und trennt unter sägenden Zügen die den Hammergriff mit der Labyrinthwand verbindenden Verwachsungen. Handelt es sich nur um strangförmige Adhäsionen, so bietet dieses Verfahren keine Schwierigkeiten dar, welche nur dann vorbanden sind, wenn flächenhafte Verwachsungen vorliegen. Wenn man das Tenotom nun frei unter dem Hammergriff hervorziehen kann, also Adhäsionen nicht mehr vorhanden sein können, führt man eine an dem Ende gekriimmte Sonde, die in unserer Klinik als "Tenotomsonde" bezeichnet wird, hinter den Hammergriff und zieht denselben so weit in den Gehörgang vor, bis er senkrecht nach unten gerichter ist. Bei diesem Hervorziehen zeigt er stets eine grosse Neigung, von der Mitte abzuweichen und sich nach forn dem Margo tympanicus zu nähern; es ist deshalb rathsam, die Tenotomsonde zum Hervorziehen des Griffes in die vordere Schnittöffnung im Trommelfell einzufuhren.

Wenn wir uns anschaulich machen wollen, welche Verände- 
rungen an der Gelenkrerbindung zwischen Hammer und Amboss bei Ausfübrung unserer Operation Platz greifen, so können wir dieselben am besten an einem frischen Schläfenbein studieren, an welchem uns die Fortnahme des Tegmen tympani einen Einblick in die Paukenhöhle vou oben gestattet. Wir können dann direct jeden Act der Operation, soweit sie sich in der Paukenhöhle abspielt, mit dem Auge verfolgen. Wir sehen, wie das Tenotom die Tensorsehne durchtrennt. Die Sehnenenden liegen nach diesem Act so nahe zusammen, dass man eine Lücke zwischen ihnen gar nicht wahrnehmen und sich nur durch Hindurchschieben einer Sonde von der stattgehabten Continuitätstrennung überzengen kann. In dem Momente, wo die gekrümmte Sonde den Hammergriff in eine mehr senkrechte Lage zu bringen bemüht ist, spannt sich der obere Theil der Gelenkkapsel der Hammer-Ambossverbindung; bei stärkerem Hervorziehen des Hammergriffes in den Gehörgang reisst sie ein, und wenn schliesslich der Hammergriff in die erstrebte Stellung gebracht ist, seben wir, dass eine Subluxation des Hammer-Ambossgelenkes eingetreten ist in dem Sinne, dass der Hammerkopf seine Lage gegen die Gelenkfläche des Amboss verändert hat. Letztere ist in ihrer ursprünglichen Lage geblieben, so dass anch eine Lageveränderung des langen Ambossschenkels nicht eingetreten ist. Die genaueste Beobachtung mittelst der Lupe hat irgend eine Veränderung an der Amboss-Steigbügelverbindung nicht feststellen können. Zwisehen den Enden der durchschnittenen Tensorsehne ist jetzt eine erbebliche Diastase eingetreten. Was nun unsere Erfahrung am Lebenden anbetrifft, so verblieb der Hammergriff im weiteren Verlaufe in der Stellung; welche inm bei der Operation gegeben war. Er zeigte keine Neigung, in seine frubere Stellung zurückzukehren. Unter dem aseptischen Occlusivverbande verheilte die Trommelfellöffnung in wenigen Wochen so, dass der hervorgeholte Hammergriff zapfenförmig über das Niveau des vernarbten Trommelfelles hervorragte.

Von Bedeutung für die Function scheint es mir bei diesem Heilungsvorgange zu sein, dass die Gehörknöchelchenkette an keiner Stelle unterbrochen ist.

In folgenden $3 \mathrm{Fällen}$ haben wir das eben geschilderte Verfahren erprobt:

Fall 1. Anton Spottog, 43 Jahre, Vorarbeiter aus Freckleben bei Sandersleben. Rec. am 15. November 1894 .

Pat. will bis vor 2 Jahres stets ohrgesund gewesen sein; damals will er 
bei Gelegenheit eines starken Schneeunwetters mit Ohrensansen und sich daran anschliessender Schwerhörigkeit beiderseits erkrankt sein. Beide Symptome bestehen ohne Besserung bis heute fort. Hereditäre Belastung nicht nachweisbar.

Stat. p raes.: Kräftig gebauter Mann, Herz und Lungen gesund. Puls 64, gut gefüllt. Resp. normal. Augenhintergrund normal. Urin ohne Zucker und Eiweiss.

o to skop. Befund: Trommelfell beiderseits vermehrt concav; links Narbe hinten unten, vorn Verkalkung. Hammergriff adhärent (Siegle).

Functionsprüfung: Leise Flüstersprache links dicht am Ohr, rechts $3 \mathrm{Cm}$. Stimmgabeln in allen Tonlagen gut gehört; in den tieferen Lagen ist die Perceptionsdauer geringer als normal. Uhr beiderseits rom Knochen besser gehört als durch Luftleitung.

Ergebniss des Cathet. tubae: Blasegeräusch beiderseits. Nach Katheter keine Hörverbesserung.

Pat. wurde längere Zeit poliklinisch behandelt (Catheter, Schwitzcur), ohne jeden Erfolg.

Dann wurde zunächst nur die Synechotomie linkerseits ausgeführt mit dem Erfolg, dass das Ohrensausen sofort verschwunden war, und das Hörvermögen sofort sich wesentlich besserte bis auf 1 Meter für leise Flüstersprache.

Diese Hörverbesserung hielt nicht lange an, so dass 8 Tage nach der 1. Operation die Tenotomie und Hervorziehung des Hammergriffes ausgeführt wurde.

Reactionsloser Heilangsverlauf. Enderfolg am 4. Mărz 1895: Links Hörvermögen für leise Flüstersprache $3 / 4$ Meter. Ohrensausen links verschwunden.

8. September 1897. Patient hat sich wieder vorgestellt. Fr giebt an, intercurrent nach Schnupfen eine linksseitige Mittelohreiterung bekommen zu haben, welche das gute Gehör wieder vernichtet habe. Danach sei ebenfalls Sausen von neuem aufgetreten.

Otoskop. Befund: Wie nach Abschluss der Nachbehandlung. Flüstersprache nur noch einige $\mathrm{Cm}$. gehört. Hohe Töne links schlecht gehört. Knochenleitung links schlecht.

Fall 2. Bernhard Prange, 15 Jahre, Musikerlehrling aus Halle a. S. 28. Januar 1896. Kommt wegen Schwerhörigkeit linkerseits.

0 toskop. Befund: Links hochgradige Einziehung des Hammergriffes; centrale Verwachsung desselben mit der Labyrinthwand. Rechts Hammergriff stark retrahirt (horizontal), vorn unten Verkalkung.

Functionsprifung: Leise Flustersprache links handbreit, rechts 1 Meter. $C_{1}$ vom Scheitel nach links verstärkt. Fis ${ }_{4}$ links nicht herabgesetzt. Rinne links negativ. Uhr rom Knochen aus deutlich gehört, durch die Luft kaum.

Catheter macht keine Hörverbesserung.

2. Februar 1896. Links Syuechotomie, Tenotomie des M. tensor tympani, Hervorziehen des Hammergriffes.

13. März. Die Trommelfellöffnung ist vernarbt; der Hammergriff steht nach vorn frei in den Gehörgang hinein. Flüstersprache links mindestens 1/2 Meter.

12. September 1897. Hammergriffstellung unverändert; links Flüsterworte mindestens 1 Meter.

Fall 3. Liese K1, 9 Jahre, aus Bielefeld. Rec. 18. November 1895.

Seit Jani 1895 bemerkte Pat. Schwerhörigkeit links mit Ohrensausen. Keine Schmerzen, weder beim Beginn, noch im weiteren Verlauf. Ursache nicht bekannt, erblich nicht belastet. Hat früher viel an. Anschwellung der Gaumenmandein gelitten. Qualität des Ohrgeräusches: monotones Zischen.

otoskop. Befund: Links leichte Gefässinjection den Hammergriff entlang. Sowohl mit Siegle's Trichter als auch bei Inspection, während mittelst des Catheters Iuft in die Paukenhöhle getrieben wird, lässt sich 
eine Adhäsion des Hammergriffsendes mit der Labyrintbwand feststellen. Rechts normal.

Functionsprüfung: Links leise Flüstersprache unsicher direct, rechts 6 Meter. $\mathrm{C}_{1}$ vom Scheitel unbestimmt. Fis ${ }_{4}$ links dentlich herabgesetzt. Uhr links rom Knochen noch gat gehört.

Nach Catheter keine Hörverbesserung.

Links Tenotomie, Synechotomie, Hervorziehen des Hammergriffes.

Nach 4 Wochen ist die Trommelfellöffnung vernarbt, der Hammergriff ragt frei nach vorn über das Trommelfellniveau in den Gehörgang hinein. Keine Hörverbesserung; das Geräusch macht zeitweise Pausen und hat eine geringere Intensität. Wie es später mit dem Ohrengeränsch geworden ist, konnte nicht eruirt werden.

Ergebniss: In allen 3 Fällen, in welchen wir dieses operative Verfahren anwandten, ging die Heilung in kurzer Zeit reactionslos von Statten. Das Trommelfell vernarbte; der Hammergriff verblieb in der Stellung, welche ihm bei der Operation gegeben worden war, so dass er dann nach dem Abschluss der Heilung frei in den Gebörgang hineinragte über das Nivean des vernarbten Trommelfelles hinweg. Er hatte somit eine Stellung, die den Wiedereintritt der ihn mit dem Promontorium verbindenden Synechienbildung von vorn herein ausschloss, eine Stellung, welche es auch wahrscheinlich macht, dass eine Wiederverwachsung der Schnittenden der Tensorsehne nicht eingetreten ist.

Was den functionellen Einfluss der Operation anbetrifft, so ersehen wir aus den Krankengeschichten, dass in den ersten beiden Fällen eine nennenswerthe Hörverbesserung eingetreten ist, welche allerdings in Fall 1. später durch eine acute Eiterung nach Schnupfen fast vollkommen wieder verloren gegangen ist. In dem 3. Falle war die Operation ohne Einfluss auf das Hörvermögen. Indess war ja auch nach dem Ergebniss der Functionsprüfung (Labyrinthbetheiligung) eine Hörverbesserung nicht zu erwarten. Das subjective Geräusch wurde aber insofern giunstig beeinflusst, als es an Intensität geringer wurde und auch zeitweise Intermissionen machte. Auch in dem 1. Falle haben wir einen günstigen Einfluss auf das Ohrensausen zu constatiren, welches nach der Operation ganz verschwand, aber später (cf. Krankengeschichte) durch eine acute intercurrente Mittelohr. eiterung wiederkehrte.

Zum Schlusse mag noch einmal hervorgehoben werden, dass das eben geschilderte operative Verfahren nur unter besonders günstigen Bedingungen einen nennenswerthen functionellen Nutzen verspricht. Nur wenn die Schwerhörigkeit allein durch die Synechie des Hammers bedingt ist, und keine anderen Compli- 
140 XI. GRUNERT, Ein neues operatives Verfahren zur Verhütung u. s. w.

cationen vorliegen, werden wir auf Erfolg rechnen können. Leider sind wir bisher nicht im Stande, durch unsere Untersuchungsmethoden alle Complicationen, welche den Effect unseres operativen Eingriffes von vornherein za einem illusorisehen machen können, diagnostisch auszuschliessen, so z. B. Veränderungen am runden Fenster, dessen Integrität doch eine Conditio sine qua non des Erfolges ist. Wenn wir uns unter diesen Verhältnissen auch nicht verhehlen können, dass diese functionellen Operationen im Mittelohr selbst bei völliger Intactheit des schallpercipirenden Apparates nur den Werth probatorischer Eingriffe haben, so hoffe ich doch, durch meine Worte die Facheollegen anregen zu können, in geeignet scheinenden Fällen diese Operationsversuche nachzuprüfen. 